\title{
FTIR-spectrophotometric analysis of levosulpiride and its pharmaceutical formulations
}

\author{
Sruthi .K ${ }^{1}$, Prasanth S.S ${ }^{2, *}$, Kamarunnisa .K ${ }^{3}$, Drisya N.K. ${ }^{4}$, Ashyam .K ${ }^{5}$ \\ 1,3,4,5Research Scholar, ${ }^{2}$ Professor, Dept. of Pharm Analysis, Al Shifa College of Pharmacy, Kizhattoor, Kerala, India
}

*Corresponding Author: Prasanth S.S

Email: nakulprasanth@gmail.com

\begin{abstract}
A fourier transform infrared (FTIR) spectrometric method was developed for the rapid, direct determination of levosulpiride in different pharmaceutical products. ${ }^{1-3}$ The universal ATR spectra were recorded and used for this study. Multiple linear regression (MLR) where a restricted set of absorption band is used for calibration beer-lambert law was used for data processing. A recovery of $98.5 \%$ of levosulpiride from the tablet dosage form with a correlation coefficient of 0.996 was obtained. The aim of this study was to develop a FTIR spectrophotometric procedure for the analysis of levosulpiride in raw material and tablets. The linear regression equation for levosulpiride was calculated to be $\mathrm{y}=189.04+152.39 * \mathrm{x}$ where $\mathrm{x}$ and $\mathrm{y}$ are concentration and integrated peak area respectively. The method had excellent reproducibility for the standard of $50 \mathrm{mg}, 49.5$ $\pm 1.02 \mathrm{mg}(\mathrm{n}=6)$. The recovery test is an experimental design to verify the relationship between the amount of substance added and the amount quantified by this assay. In this test the observed concentrations of pure levosulpiride in the powdered tablets were not significantly different from the stated concentrations by student's test, $\mathrm{p}=0.05 \%(100.04 \pm 1.49 \%, \mathrm{n}=3)$ the method gave rise to linear data in the range $2-50 . \mathrm{Mg}$ with accuracy and precision in the range $0.77-3.2 \%$. Therefore, this FTIR-spectrophotometric assay was accurate, and may be recommended for the simple quantification of levosulpiride.
\end{abstract}

Keywords: FTIR, Levosulpiride, ZNSE, AUC.

\section{Introduction}

Levosulpiride is chemically $\mathrm{N}-[(1-$-Ethylpyrrolidin2-yl) methyl]-2-methoxy5-sulfamoylbenzamide ${ }^{1}$ (Fig. 1). It is a substituted benzamide antipsychotic reported to be a selective antagonist of dopamine $\mathrm{D}_{2}$ receptor receptor with activity on both central and peripheral levels. ${ }^{2}$

It is an atypical neuroleptic and a prokinetic agent. ${ }^{3}$ Levosulpiride is also claimed to have mood elevating properties at low doses. ${ }^{4}$ Levosulpiride increases dopaminergic neurotransmission, primarily by the blocking of the dopamine autoreceptors, which inhibits the pre-synaptic dopamine synthesis and release of dopamine. Compared with racemic and dextroforms, the levo-form of sulpiride has greater central anti- dopaminergic activity, ${ }^{5}$ antiemetic, dyspeptic effects and lower acute toxicity.

Literature survey reveals that various method for analysis of levosulpiride including HPTLC, ${ }^{6}$ HPLC $^{7}$ and UV spectrophotometer. ${ }^{8-9}$ But this method proves to be more complex and is uneconomical. Here we propose an additional method for the quantification of Levosulpiride in bulk as well as formulation by Fourier Transform Infrared spectrophotometer using Zinc Selenide crystal ATR method, which is simpler and produce accurate results.

Infrared spectrometry (IR) provides an useful way for the identification of drugs. However, the traditional techniques employed to obtain the IR spectra, such as alkali halides disks, mulls and thin films, are sometimes not adequate for quantitative analysis. ${ }^{10}$ Fourier Transform (FTIR) permits continuous monitoring of the spectral baseline and simultaneous analysis of different components of the same sample. ${ }^{11-12}$

FTIR spectrometry provides information about components present in formulation. Chemometric methods, such as principal component regression (PCR), Improved Principal Component Regression and partial least squares (PLS2, Multicomponent Partial Least Squares) analysis are commonly used to extract the specific information relevant to the analyte of interest from the full spectrum. Method can be validated as per ICH guidelines. ${ }^{13}$

The main objective of this work was to develop an additional method for the fast and accurate determination of Levosulpiride in commercial pharmaceutical formulations by using the Beer-Lambert law and reducing the sample pre-treatment and providing direct FT-IR measurement.

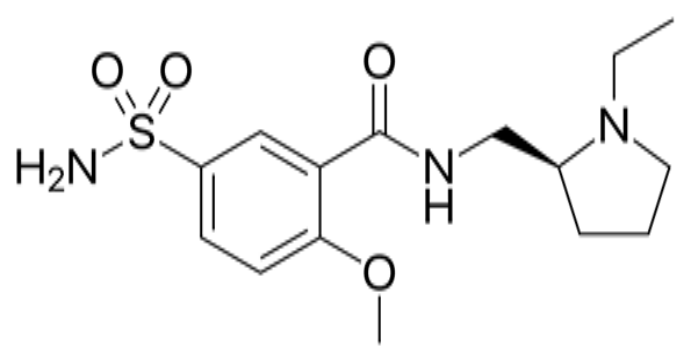

Fig. 1: Chemical structure of levosulpiride

\section{Experimental}

Apparatus: Data acquisition was performed using an FTIR spectrometer equipped with ATR, Zn-Se Crystal, OPUS Software (Bruker Co., German,). The commercial software used to generate analysis for the principal component analysis was Quant Builder.

Reagents and Materials: Levosulpiride pure sample was supplied by Sun Pharmaceuticals, Mumbai as gift sample and commercial tablets NEXIPRIDE 
manufactured by Sun Pharmaceutical Industries Ltd, Mumbai, NEOPRIDE tablets manufactured by Intas Pharmaceuticals Ltd, Ahmedabad were procured from local market.

Experimental Procedure: Accurately weighed 50mg of Levosulpiride pure drug were kept in top of ATR crystal and spectra was recorded between 4000 and 650 $\mathrm{cm}-1$, by averaging 24 scans for spectrum using OPUS software of Bruker- $\alpha$ /ZnSe FTIR spectrophotometer with Reflection Top-Plate. ${ }^{14}$ In order to compress the sample against the crystal, a pressure plate and clamp provided were used. One isolated peak 3159-3012 $\mathrm{cm}^{-1}$ was defined and peak integral area was calculated.

Linearity and Range: Different weights of Levosulpiride ranging from 50-100 mg were taken using electronic balance with $0.001 \mathrm{mg}$ sensitivity and spectrum recorded and average of such three determinations were plotted in calibration curve (Fig. 2).

LOD and LOQ: Limit of detection (LOD) and Limit of quantitation (LOQ) for the assay was calculated using the following formula: $\mathrm{LOD}=3.3 \times$ (standard deviation of $y$-intercept of the regression line / slope of the calibration curve) LOQ $=10 \times$ (standard deviation of $y$-intercept of the regression line / slope of the calibration curve)

Assay of Levosulpiride Content in Tablet Dosage form: Commercial samples of Tablet Nexipride and Neopride were taken and twenty such tablets from each company were powdered and suitable weights were taken and spectra recorded like that of standard. Peak area integration done for the same peak and quantitative analysis performed with Quant Builder option in the software.

Accuracy: Accuracy of the developed method was carried out by performing recovery study using standard addition method, in which standard drug was added at two different concentration (50\% and100\%) to the pre-analyzed formulation

Precision: Precision study of the method was performed by intra-day and inter-day variation study. The intraday precision and inter-day precision was ascertained by determining AUC of 3 replicates of a fixed concentration of the drug $(50 \mathrm{mg})$ at three different time period of the same day and on three different days. The result of the precision studies was expressed in terms of \% RSD (percentage of Relative Standard Deviation).

Reproducibility: Spectra were recorded for $50 \mathrm{mg}$ of standard drug and it was repeated for six times.

\section{Results and Discussion}

Linearity: Levosulpiride was found to be linear within the concentration range 50-100 $\mathrm{mg}$ and exhibited correlation coefficient of 0.999 (Fig. 2).

LOD and LOQ: Stacked spectra of standard Levosulpiride (Fig. 3) and overlay spectra of standards (Fig. 4) were recorded to show the precision of the method. It is of interest to note that there are no significant differences in the fingerprinting region between the spectra obtained for standard and sample, a common peak between 3159 and $3012 \mathrm{~cm}^{-1}$ was selected for Integration (Fig. 5)

Fit vs. True / Comp. 1 [mg]

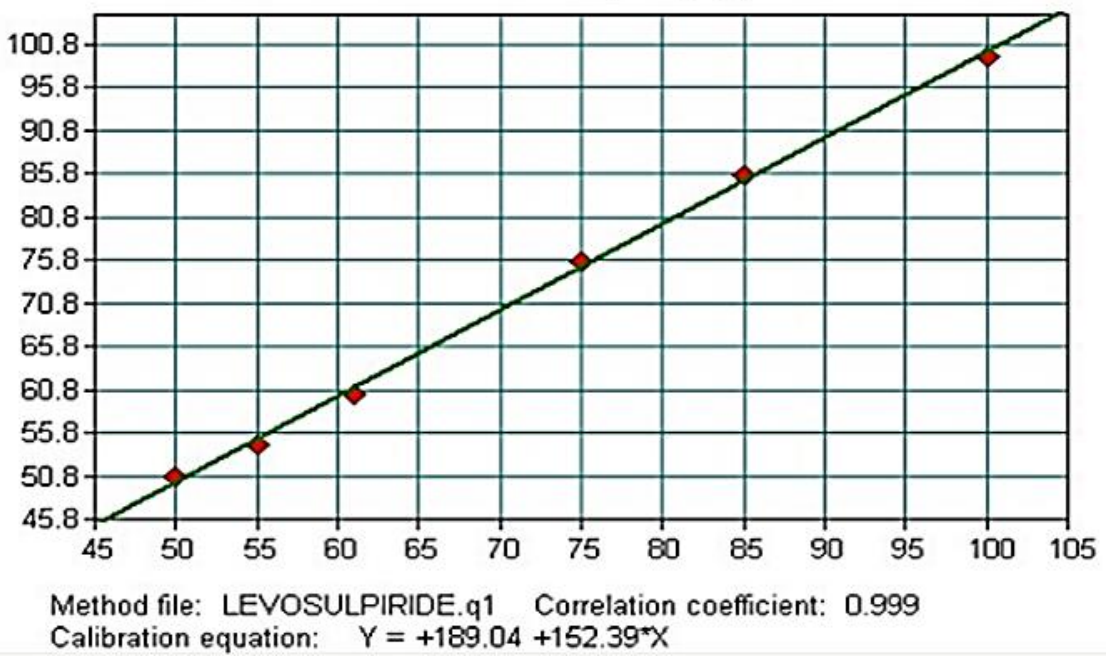

Fig. 2: Calibration graph of levosulpiride standards along with sample according to beer-lambert's law 


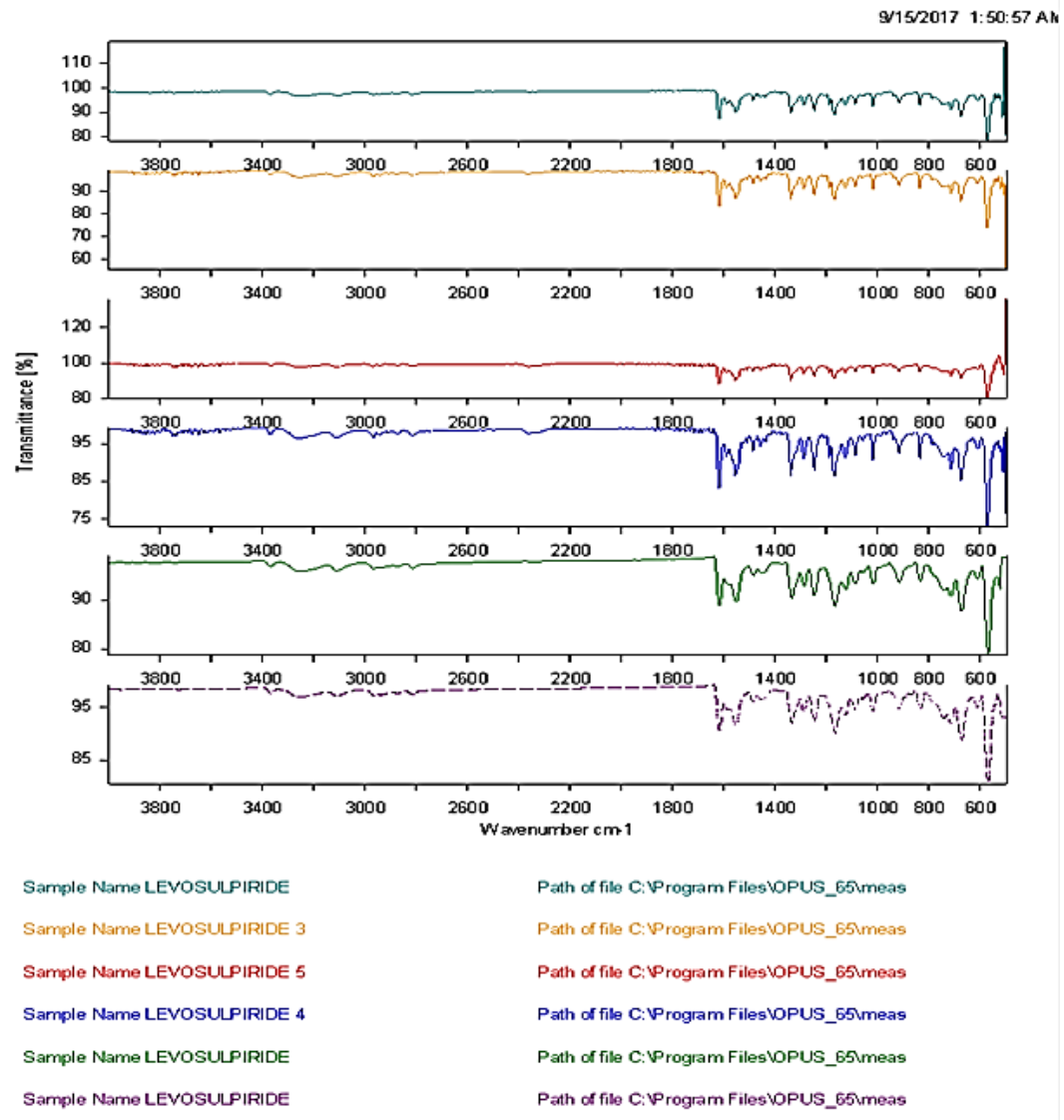

Fig. 3: Stacked spectra of levosulpiride standards

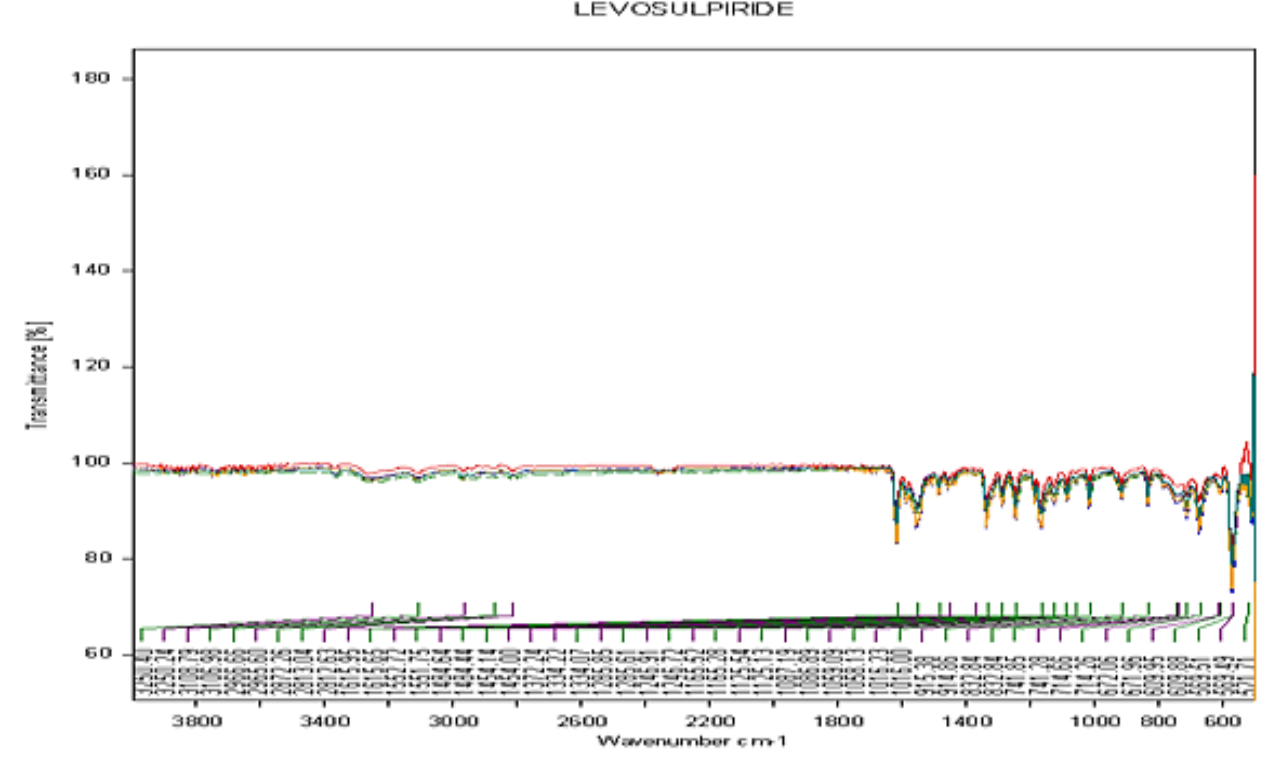

EXPeimom POSTER 1ATR_ZNSE.XPM

Path of File C:Program Filestopus_65moas

Operat or Name Administrator

Date of Measurement $01 / 01 / 2002$

Instrument Type Alpha

Sample Form POWDER

Resolution 4

Sarple Scans 24

Fig. 4: Overlay spectra of levosulpiride standards 


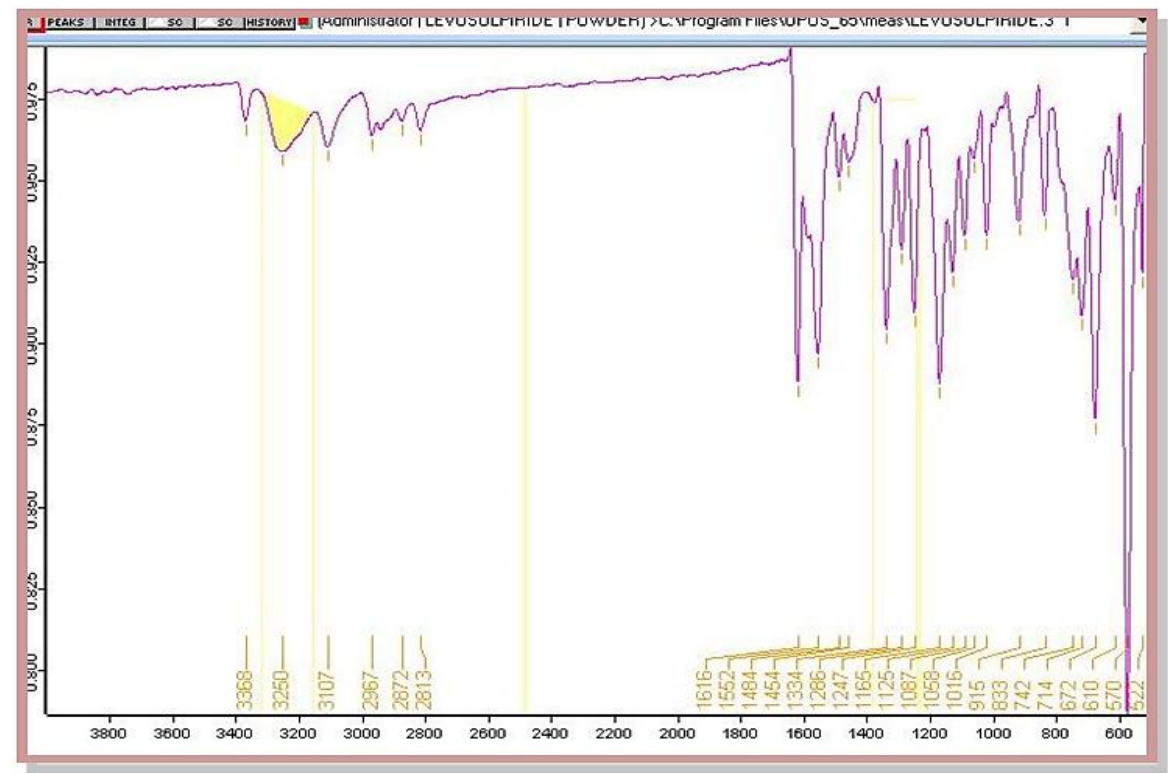

Fig. 5: Integration of standard levosulpiride

Statistical studies like Linearity, reproducibility, correlation coefficient, student's t-test, accuracy and precision were recorded. (Table 1)

Table 1: Statistical analysis

\begin{tabular}{|l|l|}
\hline \multicolumn{1}{|c|}{ Linear regression } & $\mathrm{y}=+189.04+152.39 * \mathrm{x}$ \\
\hline $\begin{array}{l}\text { Reproducibility } \\
\text { For 50 mg }\end{array}$ & $49.5 \pm 1.02 \mathrm{mg}(n=6)$. \\
\hline Student's t test & $P=0.05 \%(100.04 \pm 1.49 \%, n=3)$ \\
\hline Accuracy and precision in the range & $0.77-3.2 \%$ \\
\hline $\mathrm{R}^{2}$ & 0.999 \\
\hline Linearity & $50-100 \mathrm{mg}$ \\
\hline
\end{tabular}

Table 2: Recovery studies

\begin{tabular}{|l|c|c|c|c|}
\hline \multirow{2}{*}{ Drug } & $\begin{array}{c}\text { Amount of } \\
\text { sample } \\
\text { taken(mg) }\end{array}$ & $\begin{array}{c}\text { Amount of } \\
\text { standard } \\
\text { added(mg) }\end{array}$ & $\begin{array}{c}\text { Recovery at } \\
\text { \% }\end{array}$ & \% Recovery \\
\hline \multirow{2}{*}{ NEXIPRIDE } & 50 & 25 & 50 & 99.8 \\
\cline { 2 - 5 } & 50 & 50 & 100 & 98.6 \\
\hline \multirow{2}{*}{ NEOPRIDE } & 50 & 25 & 50 & 98.2 \\
\cline { 2 - 5 } & 50 & 50 & 100 & 99.4 \\
\hline
\end{tabular}

Table 3: Estimation of levosulpiride in tablet dosage form

\begin{tabular}{|c|c|c|c|c|c|}
\hline Drug & Sample No & $\begin{array}{c}\text { Concentration as } \\
\text { per label } \\
\text { claim(mg) }\end{array}$ & $\begin{array}{c}\text { Amount } \\
\text { found(mg) }\end{array}$ & $\begin{array}{c}\text { Percentage } \\
\text { label claim } \\
\text { \% }\end{array}$ & $\begin{array}{c}\text { Mean } \\
\text { \% }\end{array}$ \\
\hline \multirow{3}{*}{ NEXIRIDE } & 1 & 50 & 49.50 & 99.0 & \multirow{2}{*}{100.7} \\
\cline { 2 - 5 } NEOPRIDE & 2 & 50 & 51.02 & 102.4 & \\
\cline { 2 - 5 } & 1 & 50 & 49.67 & 99.30 & \multirow{2}{*}{100.45} \\
\hline
\end{tabular}

\section{Conclusions}

It is clear that FT-IR spectrometry is capable of direct determination of Levosulpiride in formulations. With the commercial software involving chemometric approaches, Beer-Lambert law, the method proposed is
Recovery studies at 50 and $100 \%$ with two tablets were found out and recorded (Table 2). An average of 98.8\% of standard has been recovered. Amount of Drug present in the two formulations were also recorded. (Table 3) 


\section{References}

1. The Merck Index an Encyclopedia of Chemicals, Drug and Biologicals, Merck \& Co. Inc. 14th edition. O'Neil M.J. 2006;1542. Editor, 2006.

2. Sweetman SC, 2007. Martindale the Complete Drug Reference, Pharmaceutical Press, London.

3. Craig RC, Robert ES. 1977. Pharmacology with Clinical Applications, Philadelphia: Lippincott Williams \& Wilkins.

4. Mucci A, Nolfe G, Maj M. Levosulpiride: A review of its clinical use in psychiatry. Pharmacol Res. 1995;31:95101.

5. Tonini M, Cipollina L, Poluzzi E, Crema F, Corazza G, De Ponti F.Clinical implications of enteric and central D2 receptor blockadeby antidopaminergic gastrointestinal prokinetics. Alimentary Pharmacology and Therapeutics. 2004;19:379-90.

6. Parmar G, Naik K, Shah S, Chauhan R \& Shah $\mathrm{D}$, Development and Validation of Stability Indicating HPTLC Method for the Estimation of Levosulpiride in Tablet Dosage Form, Analytical Chemistry Letters. 2013;3:5(6), 335-341

7. Manjunath S., Chouhan V., S. Sandeep Spectrophotometric Estimation of Levosulpiride in Bulk Drug and Formulations. International Journal of Pharmacy and Pharmaceutical Sciences. 2011;3(2):135137.
8. Silambarasan S P, Anandakumar K, Venkatalakshmi R, Sasikal C. Development of UV Spectrophotometry and RP-HPLC methodsfor the estimation of Levosulpiride in bulk and in tablet formulation. Asian J Res Chem. 2010;03(3):542.

9. Rana M, R Hasumati. A Review on Analytical Methods for Determination of Levosulpiride in Pharmaceutical Dosage Forms and Biological Sample; Pharma Tutor. 2014;2(11):66-74.

10. Andre A, FTIR of Acetyl Salicylic acid. Canadian Journal of Analytical sciences and spectroscopy. 2006,86:34-37.

11. Ciurczak E.W, Pharmaceutical and medical applications of Near-Infrared Spectroscopy, Marcel Dekker 2001;12:73-105.

12. McClure. W.F,,Analysis Using Fourier transforms in handbook of Near-Infrared Analysis. 1992;181-274.

13. International Conference on Harmonization of technical requirements for registration of pharmaceuticals for human use. Validation of Analytical Procedures: Text and Methodology. 2005;ICH Q2 (R1).

14. Wood C, Alwati A, Halsey S, Gough T, Brown E, Kelly A, Paradkar A. Near infrared spectroscopy as a multivariate process analytical tool for predicting pharmaceutical co-crystal concentration. J Pharm Biomed Anal. 2016;129:172-181. 